\title{
Down-regulated aquaporin 5 inhibits proliferation and migration of human epithelial ovarian cancer $3 \mathrm{AO}$ cells
}

\author{
ChunXiao Yan ${ }^{1}$, Yunshan Zhu' ${ }^{2}$ Xiao Zhang ${ }^{2}$, Xuejun Chen ${ }^{1}$, Wei Zheng ${ }^{1}$ and Jianhua Yang ${ }^{2 *}$
}

\begin{abstract}
Background: Recent studies suggested that aquaporins 5 (AQP5) was associated with many kinds of cancers and regulated many processes of various kinds of cancer cells. Our previous studies also demonstrated that AQP5 was highly expressed in epithelial ovarian cancer and contributed to the progress of ovarian cancer.

Methods: Lentivirus for knocking-down the expression of AQP5 was prepared and verified by qPCR and Western blotting. Cell counting kit-8 (CCK8) assay and transwell assay were performed to investigate the role of AQP5 on proliferation and migration of $3 \mathrm{AO}$ cells. The effects of down-regulating AQP5 on tumorigenesis were tested by tumor xenografts experiments.

Results: An effective lentivirus silencing AQP5 expression was obtained and used in this study. Down-regulating AQP5 inhibited proliferation and migration of cultured human epithelial ovarian cancer $3 \mathrm{AO}$ Cell. Furthermore, interfering of AQP5 during tumorigenesis could efficiently decrease the tumor growth in athymic mice.

Conclusions: These findings altogether suggest that AQP5 regulated multi processes in ovarian carcinogenesis and may be an attractive therapeutic target.
\end{abstract}

Keywords: 3AO cell, Aquaporins 5, Migration, Proliferation, Tumorigenesis

\section{Background}

Ovarian cancer is a very serious cancer. It is estimated that there will be 21,980 ovarian cancer cases newly diagnosed and 14,270 deaths due to ovarian cancer among female in the United States in 2014 which suggested that ovarian cancer will be the eleventh common cancer and the fifth common cause of cancer death in women [1]. Early stage ovarian cancer has a good 5 year survival rate at approx. $92 \%$, although $<30 \%$ of patients will present with early stage disease. The majority of ovarian cancers present at late stage disease (5 year survival $30 \%$ ) as the clinical symptoms of ovarian cancer in the early stages are vague $[2,3]$.Tumor-debulking surgery combined with following plantinum-taxane combination chemotherapy has been the fist-line treatment for ovarian cancer for over twenty year with very high response rate in the initial stage

\footnotetext{
* Correspondence: yjh0121@126.com

2Department of Obstetrics \& Gynecology, Sir Run Run Shaw Hospital affiliated to the Zhejiang University School of Medicine, Hangzhou, Zhejiang Province 310016, China

Full list of author information is available at the end of the article
}

of therapy, while most patients with advanced-stage ovarian cancer relapse within 18 months and finally die from the disease [4].

Aquaporins (AQPs) are a family of small integral plasma membrane proteins expressed in all living organisms [5]. AQPs primarily transport water and in some cases, a wide range of nonpolar solutes such as urea and glycerol and even some unconventional molecules such as the nonpolar gases carbon dioxide and nitric oxide [6]. In humans, there are 13 kinds of Aquaporins members divided into three subgroups as water selective AQPs, aquaglyceroporins and superaquaporins [7]. Aquaporins are found to mainly function as molecular transporters and involve in many disease progresses such as various chronic kidney disease, systemic disease and cancers [7-10]. Recent studies suggest that Aquaporins could be a promising drug target $[11,12]$.

The human AQP5 gene encodes a protein of 265 amino acids containing six transmembrane domain and five connecting loops [13]. Previous studies indicated that AQP5 
protein localized on the membrane of different kinds of cells such as corneal epithelium cells, acinar cells in the lacrimal gland and pulmonary epithelial cells [14]. Overexpression of AQP5 have been reported to be associated with many kinds of cancers such as colorectal, cervical, lung, breast and epithelial ovarian cancer, and to be a potential prognostic biomarkers [15-19]. The role of AQP5 in carcinogenesis is not yet fully understood. Many studies demonstrated that overexpression of AQP5 result in the increase of proliferation, migration and invasion of many kinds of human cancer cell lines [16,20-23]. Phosphorylation of AQP5 by protein kinase A (PKA) has been identified to influence the Ras activity which in turn regulated the proliferation and transformation of NIH3T3 cells [24]. Besides, the NF-kappaB signaling pathway was also proved to have a positive correlation with AQP5 expression in CAOV3 cells [25]. Our previous studies have shown that the expression of AQP5 in malignant and borderline epithelial ovarian tumors is significantly higher than that in benign tumors and normal ovarian tissue [26]. The role of AQP5 in epithelial ovarian tumorigenesis has not previously been clearly evaluated. Therefore, in this study, we used lentivirus mediated RNA interference method to down-regulate the expression to investigate the possible function of AQP5 in epithelial ovarian cancer cells.

\section{Materials and methods}

\section{Animals}

The 6-8 weeks old female BALB/c nude mice purchased from Shanghai Laboratory Animal Center, CAS serves were used to generate the tumor xenografts model. All animals were used and cared for following the Animal Research Advisory Committee of the Shanghai Institute for Biological Sciences.

\section{Generation of shRNA plasmids}

The target sequences against human AQP5 mRNA (GeneBank ID: NM_001651.3) were designed by Invitrogen Block-iT RNAi Designer, a RNAi design software of
Invitrogen Corporation. The nucleotide sequences of four candidates were shown in the Table 1. The oligonucleotides were synthesized by Invitrogen Corporation. The annealed nucleotides were then ligated into the linearized pcDNA6.2-GW/EmGFP-miR vector (Invitrogen). All constructs were sequenced for verifying.

\section{Cell cultures and transfection}

The human epithelial ovarian cancer $3 \mathrm{AO}$ cells and human embryonic kidney (HEK) 293 T cells were obtained from the Cell Bank of the Chinese Academy of Sciences (Shanghai, China) and maintained in Dulbecco modified Eagle's medium (DMEM, Gibco) containing 10\% fetal bovine serum (FBS, Gibco) and antibiotics at $37^{\circ} \mathrm{C}$ with $5 \%$ $\mathrm{CO}_{2}$. Plasmids were transfected into HEK293T cells and $3 \mathrm{AO}$ cells using Lipofectamine 2000 reagent (Invitrogen) according the manufacturer's protocol for virus package and knock-down efficiency test, respectively.

\section{Reverse-transcription PCR and qPCR}

To verify the knock down efficiency of AQP5 shRNA, $3 \mathrm{AO}$ cells were treated with Trizol reagent (Invitrogen) to extract total RNA. RNA was then reverse transcribed by M-MLV transcriptase (Invitrogen) following the manufacturer's protocol and amplified using SYBR Ex Taq premix (Takara, Japan) by iQ5 multicolor real-time PCR detection system (Bio-Rad). Primers used for qPCR were as follows: human beta-actin (ACTB) (forward primer: 5' - TCCTTCCTGGGCATGGAGT -3'; reverse primer: 5' - CAGGAGGAGCAATGATCTTGAT -3'); human AQP5 (forward primer: 5' - CTGTCCATTGGCCTGTCTGTC -3'; reverse primer: 5' - GGCTCATACGTGCCTTTGATG -3').

\section{Western blotting}

Lysates from $3 \mathrm{AO}$ cells and tissues were prepared in icecold RIPA buffer (Beyotime) supplied with protease inhibitor cocktail (Roche) and $1 \mathrm{mM}$ PMSF (Calbiochem). After centrifuge at $13,000 \times \mathrm{g}$ for $10 \mathrm{~min}$, the supernatant of lysates was denatured, loaded into the wells of the $12 \%$

Table 1 Sequence of oligonucleotides used in this study

\begin{tabular}{|c|c|}
\hline Oligonucleotides & Sequence \\
\hline shRNA-NC-F & 5'-TGCTGAAATGTACTGCGCGTGGAGACGTTTTGGCCACTGACTGACGTCTCCACGCAGTACATT-3' \\
\hline shRNA-NC-R & 5'-CCTGAAATGTACTGCGTGGAGACGTCAGTCAGTGGCCAAAACGTCTCCACGCGCAGTACATTTC-3' \\
\hline shRNA-1\#-F & 5'-TGCTGCAGTGAAGTAGATTCCGACAAGTITTGGCCACTGACTGACTTGTCGGACTACTTCACTG-3' \\
\hline shRNA-1\#-R & 5'-CCTGCAGTGAAGTAGTCCGACAAGTCAGTCAGTGGCCAAAACTTGTCGGAATCTACTTCACTGC-3' \\
\hline shRNA-2\#-F & 5'-TGCTGAACCGATTCATGACCACCGCAGTTTGGCCACTGACTGACTGCGGTGGATGAATCGGTT-3' \\
\hline shRNA-2\#-R & 5'-CCTGAACCGATTCATCCACCGCAGTCAGTCAGTGGCCAAAACTGCGGTGGTCATGAATCGGTTC-3' \\
\hline shRNA-3\#-F & 5'-TGCTGATTGAGCGGTGCCACACCGTAGTTTGGCCACTGACTGACTACGGTGTCACCGCTCAAT-3' \\
\hline shRNA-3\#-R & 5'-CCTGATTGAGCGGTGACACCGTAGTCAGTCAGTGGCCAAAACTACGGTGTGGCACCGCTCAATC-3' \\
\hline shRNA-3\#-F & 5'-TGCTGATCAGCTCCACCACCATGGCCGTTTTGGCCACTGACTGACGGCCATGGGTGGAGCTGAT-3' \\
\hline shRNA-3\#-R & 5'-CCTGATCAGCTCCACCCATGGCCGTCAGTCAGTGGCCAAAACGGCCATGGTGGTGGAGCTGATC-3' \\
\hline
\end{tabular}


sodium dodecyl sulfate polyacrylamide gel electrophoresis (SDS-PAGE) gel. The proteins were separated by electrophoresis, transferred to nitrocellulose membranes (Millipore), blocked with 5\% nonfast milk in Tris buffered saline (TBS) buffer with $0.05 \%$ Tween-20 for 1 hour at room temperature, and then probed with mouse antiAQP5 antibody (1:1300, Abcam, UK) at $4^{\circ} \mathrm{C}$ overnight. After incubation for 2 hours with horseradish-peroxidase
(HRP)-conjugated rabbit anti-mouse secondary antibody (1:5000, Santa Cruz Biotechnology, USA), bands were visualized by ECL plus western blot detection reagent (GE, USA). The house-keeping gene GAPDH was used as a loading control and detected by using mouse antiGAPDH antibody (1:4000, abmart, China) plus HRPconjugated goat anti-mouse secondary antibody (1:5000, Santa Cruz Biotechnology, USA).

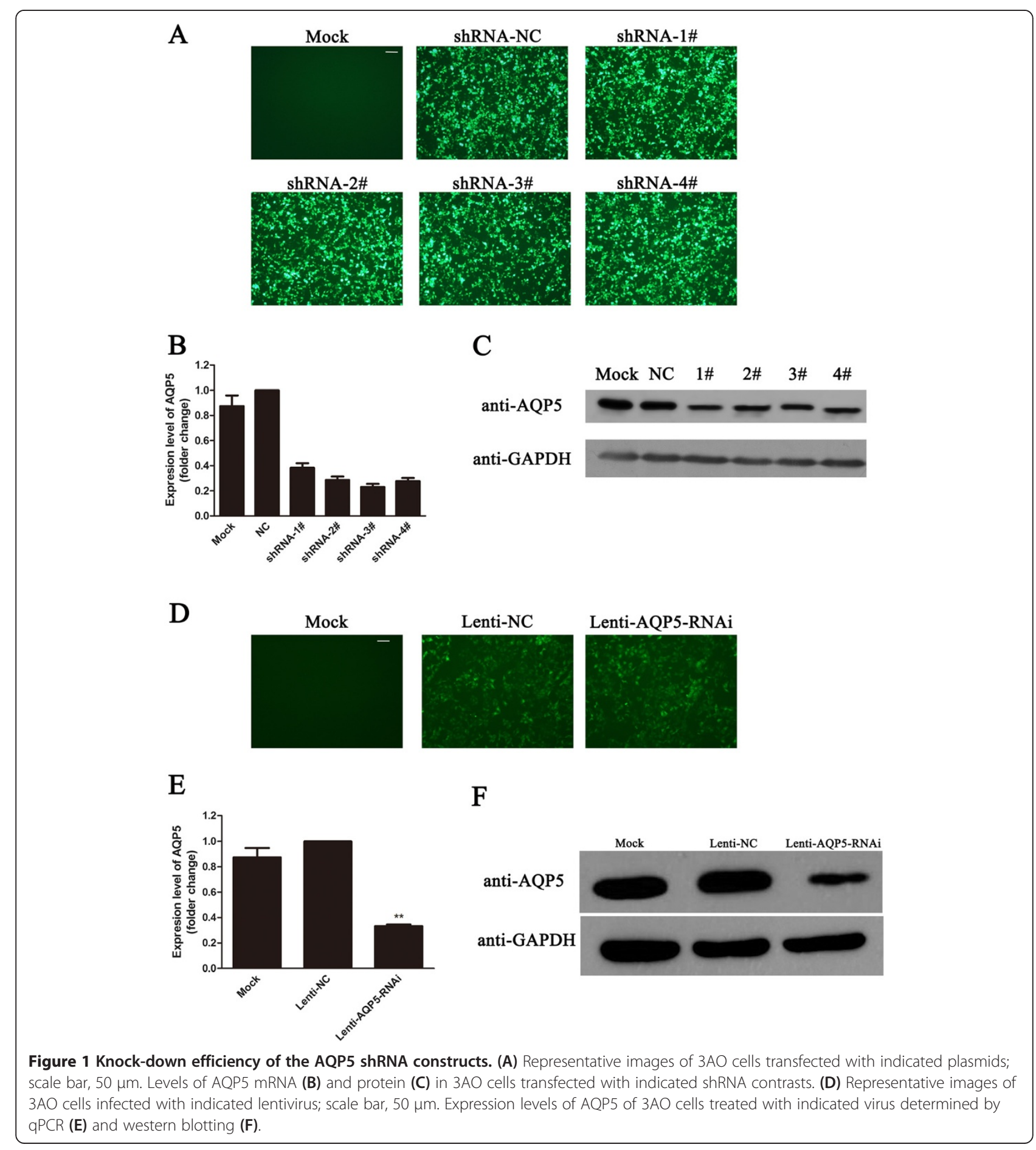




\section{Construction of AQP5 shRNA lentivirus vector and cell infection}

The most effective AQP5 shRNA tested by qPCR and western blotting was inserted into plent6.3/V5-Dest vector (Invitrogen) respectively to generate the shRNA lentivirus vector by $\mathrm{BP}$ recombination following the standard protocol. The NC control lentivirus vector was constructed in the same way. The generated plasmids were confirmed by sequence and then named as LentiAQP5-RNAi and Lenti-NC, respectively. The lentiviral vectors and packaging vectors were then transfected into 293 T cells. Supernatants containing lentivirus expressing AQP5 shRNA or control shRNA were harvested $72 \mathrm{~h}$ after transfection and then concentrated by ultracentrifugation to purify lentivirus. The titer of lentivirus was then determined by infected 293 cells. The 3AO cells were infected with lentivirus at multiplicity of infection (MOI) of 30 plus $5 \mathrm{ug} / \mathrm{ml}$ Polybrene. The knockdown efficiency of AQP5 by infection was tested by qPCR and western blotting. Then infected cells were used for CCK8 assay and migration assay.

\section{CCK8 assay}

$3 \mathrm{AO}$ cells infected by AQP5 shRNA lentivirus or control virus were trypsinized, resuspended. Then 5000 cells seeded into 96-well plate for each well. Cell viability was evaluated with Cell Counting Kit-8 (Beyotime) according to the protocol of the manufacture at daily intervals from the next day to the sixth day after seeding. After treated with CCK 8 at $37^{\circ} \mathrm{C}$ for 1 hour, $3 \mathrm{AO}$ cells was used to measure the absorbency at $450 \mathrm{~nm}$ using a microplate reader.

\section{Cell migration assay}

The migration ability of $3 \mathrm{AO}$ cells infected for 3 days was determined using a Transwell chamber (Corning). Briefly, cells were trypsinized and then resuspended by
DMEM medium without FBS to the concentration of $2 \times 10^{6} / \mathrm{ml}$. The transwell plates $(8.0$ um pore, Corning Costar) containing $100 \mathrm{ul}$ cell suspension in the upper chamber and 650 ul DMEM medium with 10\% FBS in the lower chamber was incubated at $37^{\circ} \mathrm{C}$ in $5 \% \mathrm{CO}_{2}$ for $48 \mathrm{~h}$. Then, after removing the cells remaining on the upper surface of the filter, those cells invaded to the lower compartment were fixed with Methanol and stained with crystal violet (GenMed). Cell images were captured in 3 random fields under light microscope at $4 \times, 10 \times$ and $20 \times$ magnification. In addition, invaded cells were segregated, lysed and quantified at $570 \mathrm{~nm}$ using spectrophotometer to evaluate the amount of migrated cells.

\section{Tumor xenografts}

Twenty BALB/c nude mice were divided randomly into four groups for tumor Xenografts as mock group, PBS group, Lenti-NC group and Lenti-AQP5-RNAi group. $3 \mathrm{AO}$ cells which had been trypsinized and then suspended with DMEM medium were injected subcutaneously into the BALB/c nude mice at a dose of $4 \times 10^{6} / 200 \mathrm{ul}$ for the PBS group, Lenti-NC group and Lenti-AQP5-RNAi group. 200ul DMEM medium without cells were used as a mock control. After tumors were visible in the groups injected with cells, tumor length (L) and width (W) were measured every 3 days and the first day of measurement was marked as day 0 . Tumor volume (V) was determined using the simplified formula: $\mathrm{V}=\mathrm{LW}^{2} / 2$. Once the average diameter of tumors researched approximately 4 to $6 \mathrm{~mm}$, the mice received an injection with 50 ul solution into the tumor in multi-position every five days for four times. The PBS buffer, Lenti-NC virus solution and Lenti-AQP5-RNAi virus solution were used for PBS group and mock group, LentiNC group and Lenti-AQP5-RNAi group, respectively. Tumors were harvested five days after the fourth treatment.

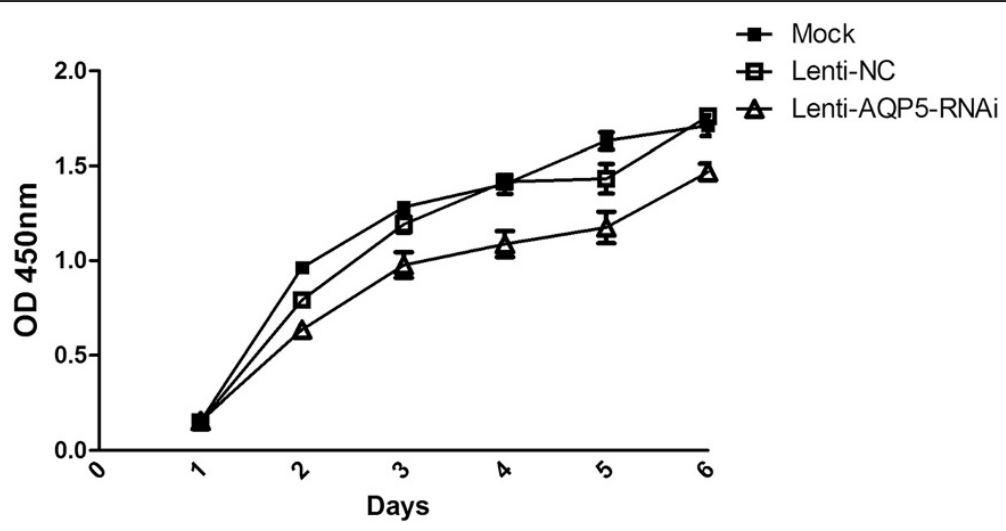

Figure 2 Knocking-down AQP5 decreases 3 AO cells proliferation. $3 A O$ cells infected with AQP5 shRNA lentivirus and various controls. The CCK8 assay demonstrated that cells treated with AQP5 shRNA have lower viability compared with the controls. The experiment was repeated for three times. Statistic significant differences were observed between cells infected with AQP5 shRNA virus and NC/mock cells. 


\section{Statistical analysis}

Statistical analysis was performed by Prism 5.0 software. Data are presented as mean \pm SEM. Significance of differences were determined by unpaired, two-tailed Student's $t$ test. $\mathrm{P}$ values $<0.05$ were considered significant.

\section{Results}

AQP5 shRNA effectively knock down AQP5 expression In order to select the most effective shRNA of AQP5, four short-hairpin RNA (shRNA) constructs that target human AQP5 mRNA and a non targeting control shRNA was transfected into $3 \mathrm{AO}$ cells, respectively. Images of transfected cell showed these constructs could be transfected into $3 \mathrm{AO}$ cells with high efficiency (Figure 1A). Both semiquantitative RT-PCR and western blotting suggested that shRNA-3\# was more effective than other shRNAs at knocking down AQP5 (Figure $1 \mathrm{~B}$ and $\mathrm{C}$ ).

The shRNA-3\# was then inserted into lentivirus vector which was used for lentiviral packaging. The concentrated lentivirus solution was used to infect $3 \mathrm{AO}$ cells. Images of infected cell suggested that $3 \mathrm{AO}$ cells could be efficiently infected by lentivirus (Figure 1D). The knocking down efficiency of Lenti-AQP5-RNAi lentivirus was proved by both semiquantitative RT-PCR and western blotting (Figure 1E and F).

\section{Down-regulation of AQP5 decrease cell proliferation of} $3 \mathrm{AO}$ cells

Our previous worked demonstrated that the high expression AQP5 was correlated with epithelial ovarian

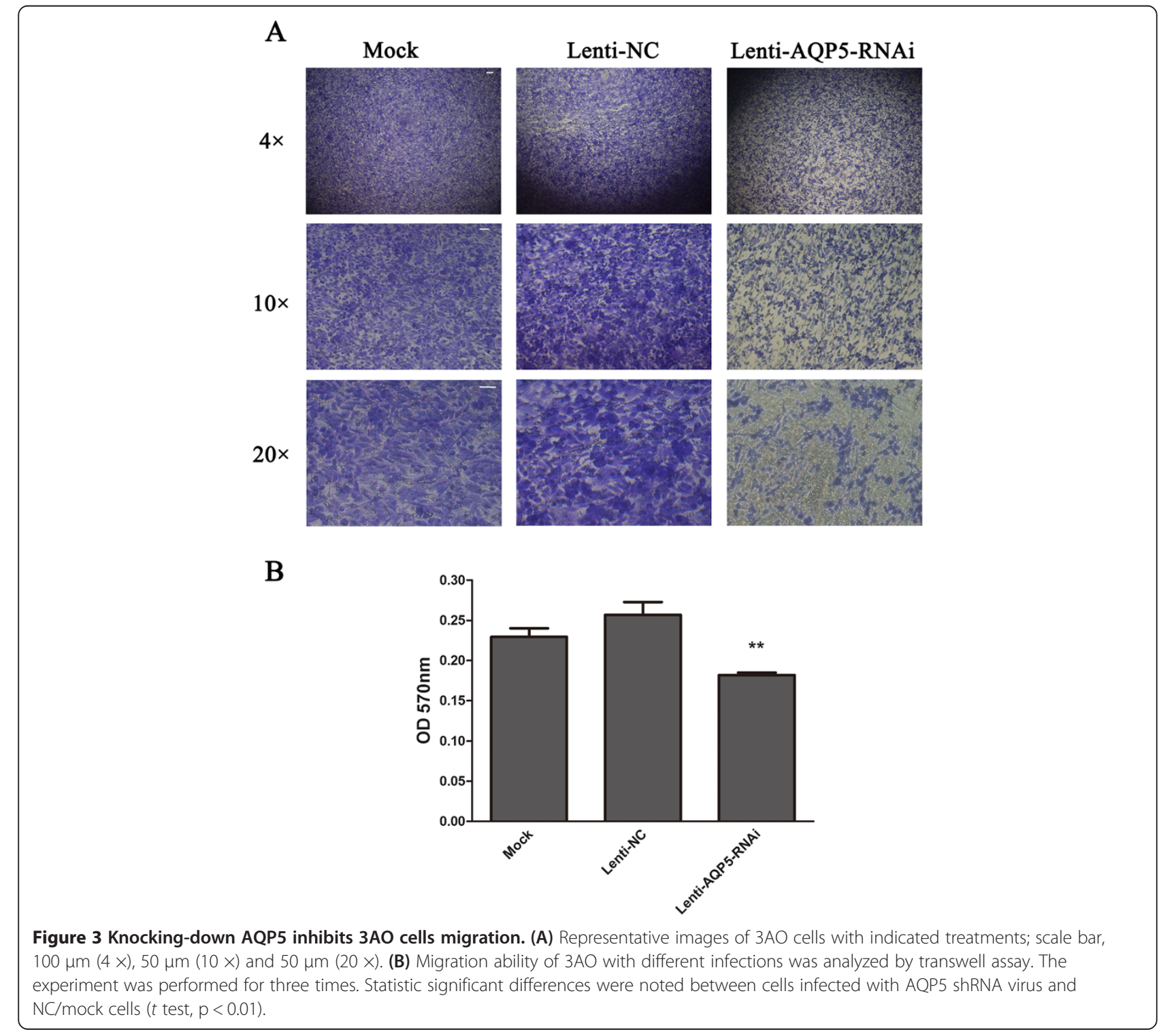


tumorigenesis [26]. To investigate the role of AQP5 in ovarian tumorigenesis, we firstly examined whether AQP5 involved in the proliferation of ovarian cancer cells. Cells infected with lentivirus or without treatment were used to measure the cell viability by CCK 8 assay for 6 days. Compared to cells without treatment or transfected with Lenti-NC virus, cells with low expression level of AQP5 had a lowed cell proliferation ability (Figure 2). This initial result suggested that AQP5 played a potential role in ovarian cell proliferation.

\section{APQ5 is involved in cell migration}

To further explore the role of AQP5 in ovarian tumorigenesis, we used Tanswell assay to investigate whether AQP5 is associate with migration ovarian cell. The Images of cells stained with crystal violet suggested that knocking down the expression of AQP5 resulted in the decrease of amount of invaded cells (Figure 3A). Additional observation of absorption assay also proved that the migration ability of $3 \mathrm{AO}$ cell reduced after knocking down the expression of AQP5 (Figure 3B). All of these data suggested that AQP5 was involved in the migration of ovarian cancer cells.

\section{Reduction expression of AQP5 inhibited tumorigenesis in tumor xenografts model}

To verify the role of AQP5 in tumorigenesis, we performed xenoplantation in athymic mice with different kinds of injection in the tumorigenesis process. The growth rate of tumor size in mice receiving Lenti-AQP5-RNAi virus started to slow down after virus treatment. While the tumor in other two treatment groups continued to grow rapidly. In contrast, mice in the mock group treated with
PBS buffer though the experiment did not form any detectable tumor (Figure 4).

\section{Discussion}

Our previous studies have identified that the expression level of AQP5 in malignant and borderline ovarian tumors is significant higher than those in benign tumors [26]. Our studies also suggested the probable relationship between NF-kappaB pathway and AQP5 expression in human ovarian cancer cells [25]. Our present report gave us more insights into the role of AQP5 in the ovarian carcinogenesis process. It suggested that AQP5 was positively associated with both proliferation and migration of ovarian cancer cells, which may also have affected the growth of tumor in the xenoplantation model. All of these preliminary findings explored the role AQP5 in ovarian cancer and indicated that AQP5 is a putative oncogene and may play multi roles in ovarian carcinoma.

The literature suggested that AQP5 paly a significant role in the cancer process and be a potential early biomarker for many kinds of tumors $[2,18,27,28]$. While the detailed function of AQP5 in cancer is still unclear. Previous studies proved that deletion of cAMP-protein kinase (PKA) phosphorylation substrate sequence of AQP5 could inhibit the cell proliferative ability of AQP5 in NIH 3 T3 cells. In addition, the PKA consensus site of AQP5 was found to be phosphorylated preferentially in tumors [20]. The following works further demonstrated that the phosphorylation of AQP5 by PKA activated Ras activity which regulated proliferation and transformation of cancer cells [24]. Thus, it is possible that a decrease of AQP5 lead to the inhibition of Ras activity and then cell proliferation which could be investigated in our ongoing studies.

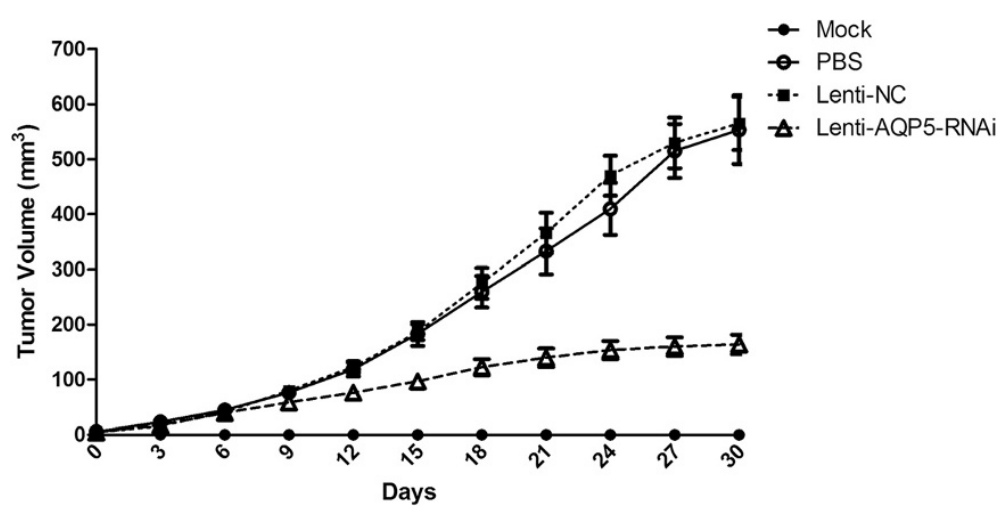

Figure 4 Down-regulating AQP5 expression appears to inhibit tumor growth in athymic mice. Tumor growth was observed within 30 days since visible tumor observed. Lentivirus solution or PBS buffer were subcutaneously injected when the average diameter of tumors researched approximately 4 to $6 \mathrm{~mm}$. No tumor growth was observed in mice injected with PBS instead of 3AO cells. Means and standard deviations of tumor volume were calculated from multiple observations in three groups of mice. Statistically significant differences were noted between mice received Lenti-AQP5-shRNA virus injection and mice injected with Lenti-NC virus or PBS buffer ( $t$ test, $p<0.01) . n=5$ per group. 
A recent discovery of the involvement of AQP5 in cell migration suggested that AQP5 may determine cell migration ability by mediating water permeability and regulating cell shape and volume [29]. Besides, the upregulation of AQP5 by E2 could increase annexin-2, a regulator of F-actin remodeling and rearrangement, and result in the increase of cell migration, invasion and adhesion in Ishikawa cells [23]. These studies helped us to understand the role of AQP5 in the cancer cell migration process. However, more direct and detailed mechanisms are needed in order to approve the work.

Inhibition of tumor growth by down regulation was also observed in tumor xenografts model. As injection of virus with shRNA target AQP5 was performed after visible tumors were generated, our observation suggested that AQP5 should play an important role in the ovarian carcinogenesis and be a potential drug target for ovarian treatment.

\section{Conclusions}

In conclusion, this report provides systemic evidence of the multi role of AQP5 in ovarian tumorigenesis and indicates that AQP5 is a promising treatment target beyond a biomarker for ovarian prognosis. Further investigations on the pathways APQ5 may involved in are need to understand detailed roles in tumorigenesis.

\section{Competing interests}

The authors state that they have no competing interest.

\section{Authors' contributions}

CY involved in the design of this study, generated the shRNA construct, performed all statistical analyses, interpreted experimental data and drafted the manuscript. YZ was in charge of cell culture, transfection, virus packaging, and virus infection. XZ participated the tumor xenografts experiments. XC, WZ and XZ contributed the GPCR, western blotting and transwell assay, respectively. JY substantially contributed to the conception and design of the study, and helped draft the manuscript. All authors have read and approved the final version of the manuscript.

\section{Acknowledgements}

This study was supported by grants of National Natural Science Foundation of China (grant number: 200904435047 \& 81202064).

\section{Author details}

'Department of Gynecology, The Second Affiliated Hospital, School of Medicine, Zhejiang University, Hangzhou, Zhejiang Province, China. ${ }^{2}$ Department of Obstetrics \& Gynecology, Sir Run Run Shaw Hospital affiliated to the Zhejiang University School of Medicine, Hangzhou, Zhejiang Province 310016, China.

Received: 4 March 2014 Accepted: 28 July 2014

Published online: 15 August 2014

\section{References}

1. Siegel R, Ma J, Zou Z, Jemal A: Cancer statistics, 2014. CA Cancer J Clin 2014, 64:9-29.

2. Su Z, Graybill WS, Zhu Y: Detection and monitoring of ovarian cancer. Clin Chim Acta 2013, 415:341-345.

3. Yurkovetsky Z, Skates S, Lomakin A, Nolen B, Pulsipher T, Modugno F, Marks J, Godwin A, Gorelik E, Jacobs I, Menon U, Lu K, Badgwell D, Bast RC Jr, Lokshin AE:
Development of a multimarker assay for early detection of ovarian cancer. J Clin Oncol 2010, 28:2159-2166.

4. Ko SY, Naora H: Therapeutic strategies for targeting the ovarian tumor stroma. World J Clin Cases 2014, 2:194-200.

5. Jeyaseelan K, Sepramaniam S, Armugam A, Wintour EM: Aquaporins: a promising target for drug development. Expert Opin Ther Targets 2006, 10:889-909.

6. Hachez C, Chaumont F: Aquaporins: a family of highly regulated multifunctional channels. Adv Exp Med Biol 2010, 679:1-17.

7. Ishibashi K, Hara S, Kondo S: Aquaporin water channels in mammals. Clin Exp Nephrol 2009, 13:107-117.

8. Schey KL, Wang Z, LWenke J, Qi Y: Aquaporins in the eye: expression, function, and roles in ocular disease. Biochim Biophys Acta 2014, 1840:1513-1523.

9. Ribatti D, Ranieri G, Annese T, Nico B: Aquaporins in cancer. Biochim Biophys Acta 2014, 1840:1550-1553.

10. Holmes RP: The role of renal water channels in health and disease. Mol Aspects Med 2012, 33:547-552.

11. Huber VJ, Tsujita M, Nakada T: Aquaporins in drug discovery and pharmacotherapy. Mol Aspects Med 2012, 33:691-703.

12. Wang F, Feng XC, Li YM, Yang H, Ma TH: Aquaporins as potential drug targets. Acta Pharmacol Sin 2006, 27:395-401.

13. Raina S, Preston GM, Guggino WB, Agre P: Molecular cloning and characterization of an aquaporin cDNA from salivary, lacrimal, and respiratory tissues. J Biol Chem 1995, 270:1908-1912.

14. Funaki H, Yamamoto T, Koyama Y, Kondo D, Yaoita E, Kawasaki K, Kobayashi H, Sawaguchi S, Abe H, Kihara I: Localization and expression of AQP5 in cornea, serous salivary glands, and pulmonary epithelial cells. Am J Physiol 1998, 275:C1151-1157

15. Yang JH, Shi YF, Cheng Q, Deng L: Expression and localization of aquaporin-5 in the epithelial ovarian tumors. Gynecol Oncol 2006, 100:294-299.

16. Chae YK, Woo J, Kim MJ, Kang SK, Kim MS, Lee J, Lee SK, Gong G, Kim YH, Soria JC, Jang SJ, Sidransky D, Moon C: Expression of aquaporin 5 (AQP5) promotes tumor invasion in human non small cell lung cancer. PLOS One 2008, 3:e2162.

17. Shi Z, Zhang T, Luo L, Zhao H, Cheng J, Xiang J, Zhao C: Aquaporins in human breast cancer: identification and involvement in carcinogenesis of breast cancer. J Surg Oncol 2012, 106:267-272.

18. Wang W, Li Q, Yang T, Bai G, Li D, Sun H: Expression of AQP5 and AQP8 in human colorectal carcinoma and their clinical significance. World J Surg Oncol 2012, 10:242.

19. Huang YH, Zhou XY, Wang HM, Xu H, Chen J, Lv NH: Aquaporin 5 promotes the proliferation and migration of human gastric carcinoma cells. Tumour Biol 2013, 34:1743-1751.

20. Woo J, Lee J, Chae YK, Kim MS, Baek JH, Park JC, Park MJ, Smith IM, Trink B, Ratovitski E, Lee T, Park B, Jang SJ, Soria JC, Califano JA, Sadransky D, Moon C: Overexpression of AQP5, a putative oncogene, promotes cell growth and transformation. Cancer Lett 2008, 264:54-62.

21. Zhang Z, Chen Z, Song Y, Zhang P, Hu J, Bai C: Expression of aquaporin 5 increases proliferation and metastasis potential of lung cancer. J Pathol 2010, 221:210-220

22. Jung HJ, Park JY, Jeon HS, Kwon TH: Aquaporin-5: a marker protein for proliferation and migration of human breast cancer cells. PLOS One 2011, 6:e28492.

23. Jiang XX, Xu KH, Ma JY, Tian YH, Guo XY, Lin J, Wu RJ: Reduced migration of Ishikawa cells associated with downregulation of aquaporin-5. Oncol Lett 2012, 4:257-261.

24. Woo J, Lee J, Kim MS, Jang SJ, Sidransky D, Moon C: The effect of aquaporin 5 overexpression on the Ras signaling pathway. Biochem Biophys Res Commun 2008, 367:291-298.

25. Yang J, Yan C, Zheng W, Chen X: Proliferation inhibition of cisplatin and aquaporin 5 expression in human ovarian cancer cell CAOV3. Arch Gynecol Obstet 2012, 285:239-245.

26. Yang $J H, Y u Y Q$, Yan CX: Localisation and expression of aquaporin subtypes in epithelial ovarian tumours. Histol Histopathol 2011, 26:1197-1205.

27. Shen $L$, Zhu Z, Huang $Y$, Shu $Y$, Sun $M, X u H$, Zhang $G$, Guo $R$, Wei W, Wu W: Expression profile of multiple aquaporins in human gastric carcinoma and its clinical significance. Biomed Pharmacother 2010, 64:313-318 
28. Frede J, Fraser SP, Oskay-Ozcelik G, Hong Y, loana Braicu E, Sehouli J, Gabra H, Djamgoz MB: Ovarian cancer: Ion channel and aquaporin expression as novel targets of clinical potential. Eur J Cancer 2013, 49:2331-2344.

29. Chen Z, Zhang Z, Gu Y, Bai C: Impaired migration and cell volume regulation in aquaporin 5-deficient SPC-A1 cells. Respir Physiol Neurobiol 2011, 176:110-117.

doi:10.1186/s13048-014-0078-2

Cite this article as: Yan et al: Down-regulated aquaporin 5 inhibits proliferation and migration of human epithelial ovarian cancer $3 \mathrm{AO}$ cells. Journal of Ovarian Research 2014 7:78.

\section{Submit your next manuscript to BioMed Central and take full advantage of:}

- Convenient online submission

- Thorough peer review

- No space constraints or color figure charges

- Immediate publication on acceptance

- Inclusion in PubMed, CAS, Scopus and Google Scholar

- Research which is freely available for redistribution 